\title{
First motor seizure as presenting symptom of SARS-CoV-2 infection
}

\author{
Antonio Fasano $^{1,2}$ (D) Francesco Cavallieri $^{1,2}$ (D) Elena Canali $^{2} \cdot$ Franco Valzania $^{2}$
}

Received: 15 April 2020 / Accepted: 7 May 2020 / Published online: 16 May 2020

(C) Fondazione Società Italiana di Neurologia 2020

Dear Sir,

The SARS-CoV-2 pandemic is currently causing concern in the medical community as the virus is rapidly spreading around the world [1]. Since its identification in late December 2019 in China, the epidemiological picture is changing on a daily basis: in the last month, Italy has been the center of the European outbreak with a growing number of infected patients. Although patients with $S A R S-C o V-2$ disease typically present with respiratory symptoms, pieces of information suggest that the virus may also have neurological manifestations [2]. We report a case of first motor seizure as presenting symptom of SARS-CoV-2 infection. The present case further underscores that initial manifestations of novel Coronavirus infection might be non-specific and it highlights the need for physicians to consider potential neurological manifestations of $S A R S-C o V-2$ infection.

\section{Case report}

On February 28, 2020, a 54-year-old firefighter was admitted to our emergency room (ER) after a single seizure characterized by clonic movements in the right arm and loss of consciousness. His

Antonio Fasano

antonio.fasano@ausl.re.it; 147325@studenti.unimore.it; antonio.fasano89@gmail.com

Francesco Cavallieri

francesco.cavallieri@ausl.re.it; francesco.cavallieri@unimore.it

Elena Canali

elena.canali@ausl.re.it

Franco Valzania

franco.valzania@ausl.re.it

1 Clinical and Experimental Medicine, University of Modena and Reggio Emilia, L.go del Pozzo, 71, 41124 Modena, Italy

2 Neurology Unit, Neuromotor \& Rehabilitation Department, Azienda USL-IRCCS di Reggio Emilia, Viale Risorgimento 80, 42123 Reggio Emilia, Italy relatives reported a sudden onset of involuntary and rhythmic movements involving the right hand and arm lasting about 1 or 2 min; after that, it was reported that the patient appeared generally stiffened and he lost consciousness. When he awakened, he was confused for about $30 \mathrm{~min}$. At the ER admission, he reported suffering from conjunctivitis in the last 10 days and mild fever in the past week. He had no remote medical or neurological history; he had been taking ophthalmic steroids during the previous days but he was not under regular medications; there was no history of drug use. Vital parameters, peripheral oxygen saturation, and EKG were normal (body temperature $36.5^{\circ} \mathrm{C}$ ); neurological examination showed no focal deficits. Serum laboratory tests revealed neutrophilia ( $85.5 \%$, NR 40-75\%), normal white blood cell count $(6.38 \times 1000 / \mu \mathrm{L}, \mathrm{NR} 4.0-10.0)$, lymphopenia $(0.69 \times$ $1000 / \mu \mathrm{L}, \mathrm{NR} 0.8-4.0)$, normal serum sodium $(140.8 \mathrm{mmol} / \mathrm{L}$, NR 132.0-146.0), slightly elevated CRP (1.29 mg/dL, NR 00.5 ), and AST and ALT enzymes (57 and $59 \mathrm{UI} / \mathrm{L}$ respectively, NR 2-40 and 4-49). Head CT scan was unremarkable (Fig. 1a). Chest X-ray showed a single lingular disventilatory band (Fig. 1b). Urinalysis test, urine culture, and urinary antigen tests for Streptococcus pneumoniae and Legionella pneumophila were normal. The patient's electroencephalogram recorded the following day showed no abnormalities (Fig. 1c). In the suspicion of Mycoplasma pneumoniae infection, Azithromycin $500 \mathrm{mg}$ QD for 3 days was prescribed. On February 29, the patient was discharged and a diagnosis of "first focal motor seizure" was made; no anti-epileptic medications were prescribed. After 4 days, on March 4, the patient was admitted to the ER again reporting cough, high fever, and shortness of breath; his daughter also had a high fever. His temperature was $38.5^{\circ} \mathrm{C}$, the heart rate was $100 \mathrm{bpm}$, and the peripheral oxygen saturation was $94 \%$. Lab tests showed raised inflammatory markers; chest X-ray pointed out the appearance of mild bilateral perihilar consolidations (Fig. 1d); chest HR-CT scan showed bilateral ground-glass opacities (Fig. 1e). Based on epidemiological data and according to the government guidelines, two nasopharyngeal swabs were obtained and were found to be positive for SARS-CoV-2 on qRTPCR. The patient was hospitalized in the Intermediate Care Unit and non-invasive ventilation (NIV) was undertaken. He 


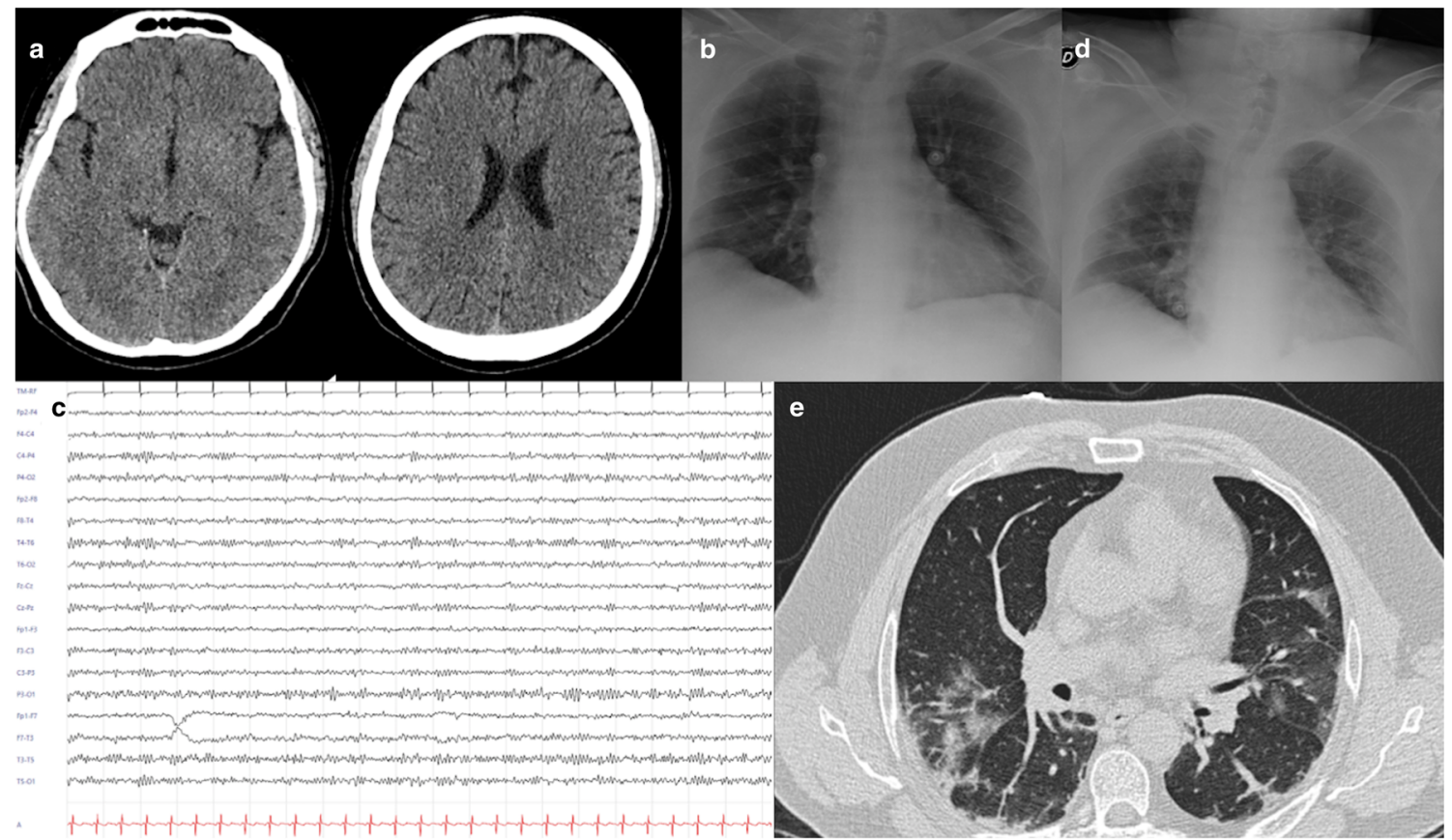

Fig. 1 Chest $\mathrm{x}$-rays, chest HRCT, brain CT scan, and EEG findings. Brain CT scan did not show any abnormalities (a). First chest x-ray performed on February 28th showed a lingular disventilatory band (b). Electroencephalogram (EEG) did not show any abnormalities (c). Second

completely recovered after 2 weeks of antiviral therapy (Lopinavir-Ritonavir); he remains seizure-free.

The consultant neurologist who had been in close contact with the patient during the first ER admission was isolated for clinical monitoring. She had no signs or symptoms of infection and she tested negative for SARS-CoV-2.

\section{Discussion}

Europe and the USA are currently facing the SARS-CoV-2 pandemic. This is primarily a respiratory disease marked by cough, fever, and shortness of breath [3]. However, it has been suggested that it might have neurologic complications in some instances. A report from a Chinese hospital pointed out that frequent neurological symptoms are altered consciousness, skeletal muscle damage, and cerebrovascular diseases [2]. We are reporting a case of a patient suffering from a first seizure as a possible presenting symptom of SARS-CoV-2 infection. The initial presentation of the novel Coronavirus can be non-specific [3]: retrospectively, the patient's initial exams (lymphocytopenia and chest Xray disventilatory band) were consistent with manifestations of $S A R S-C o V-2$ infection [3]. Considering the temporal association between seizure onset and novel Coronavirus infection, we hypothesize that this infection might have been responsible for the development of seizures in this patient. We speculate that the suggested SARS-CoV-2-induced "cytokine storm syndrome" [4] may have contributed to seizures onset, considering the chest x-ray performed on March 4th showed the appearance of mild bilateral peri-hilar consolidations (d). Chest HRCT scan carried out on the same day of the second chest x-ray revealed bilateral peripheral ground-glass opacities (GGO) compatible with interstitial pneumonia (e)

normality of both the serum sodium level and the peripheral saturation at the time of first hospitalization [5].

One limitation of this case is the absence of SARS-CoV-2 testing on the first admission. Considering the previous history of mild fever and conjunctivitis, it is likely that the patient suffered from SARS-CoV-2 infection even at the time of the first hospital admission. Secondly, the differential diagnosis included a cardiac syncope: our patient, however, did not suffer from any heart diseases, he was not performing any physical activity at the time of seizure onset, and he had a long recovery time when he awakened; moreover, his EKG and blood pressure were normal during the first hospital admission. Finally, we have not yet performed a brain MRI. The patient's neurological examination, however, was normal both before and after the seizure with no clinical deficits possibly related to brain lesions; the brain CT, moreover, showed no focal abnormalities.

In conclusion, this case only suggests a possible association between seizures onset and SARS-CoV-2 infection and it points out the need to consider potential neurological symptoms of the novel Coronavirus; further cases with epidemiological data will clarify if the association is accidental or causative. This case also highlights the need for clinicians to be alert to $S A R S-C o V-2$ neurological complications. This is important both for patients and for neurologists. On the one hand, patients need to be quickly and accurately diagnosed in order to avoid delayed diagnoses and to prevent further transmission; on the other hand, neurologists must be protected from the risk of contracting the SARS-CoV-2 infection even in non-specific situations. 
Authors' contributions - Antonio Fasano: Design and conceptualized study; major role in the acquisition of data; analyzed the data; drafted the manuscript for intellectual content.

- Francesco Cavallieri: Major role in the acquisition of data; interpreted the data; revised the manuscript for intellectual content.

- Elena Canali: Acquisition of data; interpreted the data; revised the manuscript for intellectual content.

- Franco Valzania: Design and conceptualized study; interpreted the data; revised the manuscript for intellectual content.

All authors have approved the final article.

Data availability Dr. Fasano as the corresponding author has full access to all the data (data transparency).

\section{Compliance with ethical standards}

Conflict of Interest The authors declare that they have no conflict of interest.

Ethical approval Not applicable.

Consent to participate Not applicable.

Consent for publication Written informed consent was obtained from the patient for publication.

Informed consent The patient gave his informed consent for case description.

Code availability Not applicable.

\section{References}

1. Zhu N, Zhang D, Wang W, Li X, Yang B, Song J, Zhao X, Huang B, Shi W, Lu R, Niu P, Zhan F, Ma X, Wang D, Xu W, Wu G, Gao GF, Tan W, China Novel Coronavirus Investigating and Research Team (2020) A novel coronavirus from patients with pneumonia in China, 2019. N Engl J Med 382:727-733. https://doi.org/10.1056/ NEJMoa2001017

2. Mao L, Jin H, Wang M, Hu Y, Chen S, He Q, Chang J, Hong C, Zhou Y, Wang D, Miao X, Li Y, Hu B (2020) Neurologic manifestations of hospitalized patients with coronavirus disease 2019 in Wuhan, China. JAMA Neurol. https://doi.org/10.1001/jamaneurol. 2020.1127

3. Guan W, Ni Z, Hu Y, Liang WH, Ou CQ, He JX, Liu L, Shan H, Lei CL, Hui DSC, du B, Li LJ, Zeng G, Yuen KY, Chen RC, Tang CL, Wang T, Chen PY, Xiang J, Li SY, Wang JL, Liang ZJ, Peng YX, Wei L, Liu Y, Hu YH, Peng P, Wang JM, Liu JY, Chen Z, Li G, Zheng ZJ, Qiu SQ, Luo J, Ye CJ, Zhu SY, Zhong NS, China Medical Treatment Expert Group for Covid-19 (2020) Clinical characteristics of coronavirus disease 2019 in China. N Engl J Med. 382:17081720. https://doi.org/10.1056/NEJMoa2002032

4. Mehta P, McAuley DF, Brown M et al (2020) COVID-19: consider cytokine storm syndromes and immunosuppression. Lancet 395: 1033-1034. https://doi.org/10.1016/S0140-6736(20)30628-0

5. Esen F, Orhun G, Özcan PE, Brenes Bastos AR, Tüzün E (2020) Diagnosing acute brain dysfunction due to sepsis. Neurol Sci 41:2533. https://doi.org/10.1007/s10072-019-04069-x

Publisher's note Springer Nature remains neutral with regard to jurisdictional claims in published maps and institutional affiliations. 